\title{
Research on Indoor Localization Algorithm Based on SVM
}

\author{
Gao Xin1)
}

\begin{abstract}
In order to solve the problem of a large positioning error, low refresh rate and high-power consumption of UWB in an indoor high-density environment. This paper proposes a UWB indoor positioning method based on SVM (support vector machine), which transforms the positioning problem into the solution of the multi-classification problem. The SVM model based on TDOA (time difference of arrival) is established, and the one-to-one classification model is used to solve the coordinate value quickly. Experimental results show that the proposed algorithm has higher accuracy and less time in indoor positioning. The algorithm proposed in this paper has more application prospects.
\end{abstract}

Keywords: Support Vector Machine, UWB, Indoor Positioning Method, TDOA

\section{Introduction}

With the development of LBS (location-based services), people demand higher and higher positioning accuracy in dry location services. UWB (ultra-wide band) transmits data through narrow pulses of non-sinusoidal waves ranging from nanoseconds to microseconds. One of the salient features of UWB is its high data transmission rate. In addition, UWB also has strong anti-interference ability, fast transmission rate, large system memory, good penetration performance, small power transmission and other characteristics. It stands out among many wireless positioning technologies[1], so UWB technology can realize high-precision indoor positioning and provide accurate positioning navigation for location services.

Since TOA, time of arrival, is mainly based on the measurement of arrival time transmitted between nodes, TOA has strict requirements on time synchronization of each node. In addition, the TOA positioning algorithm requires at least three times of two-way communication between the base station and the mobile station for distance measurement, which is also the main reason why the power loss of the base station and the mobile station increases greatly. The TDOA (time difference of arrival) positioning technique requires only precise time synchronization in the receiver, not absolute time synchronization between the receiver and the

Received(October 18, 2019), Review Result(1st: November 07, 2019, 2nd: December 13, 2019), Accepted(February 20, 2020)

1) (Professor) Harbin university of commerce, Harbin, Heilongjiang province, China email: zz191i@126.com 
sender. Therefore, clock accuracy is easier to achieve than TOA positioning method[2]. Therefore, TDOA is more widely used. Traditional TDOA localization algorithms include Chan algorithm, Fang algorithm, Taylor algorithm and so on. Chan algorithm[3] has high positioning accuracy in the case of LOS, line of sight or when the measurement error of TDOA is small. In the case of NLOS (not line of sight) or when the channel performance is relatively poor, the positioning accuracy will greatly decline, so one of the important factors affecting the positioning accuracy is the non-line-of-sight error. Taylor series expansion method[4] is a kind of recursive algorithm. This method needs to estimate the initial position, and the computational complexity is relatively large. The estimated value of the initial position has a great impact on the positioning accuracy. Fang algorithm[5] can only use three base stations for positioning, and the TDOA measurement values given by other base stations are not fully utilized. The simulation results of literature[6] show that in the actual UWB positioning, Chan and Taylor algorithms are two algorithms with relatively high positioning accuracy.

Support vector machine (SVM) is often used for voice recognition, biological medicine, noise processing[7], etc. Support vector machines (SVM) can solve high dimensional problems and machine learning problems with small samples. SVM can deal with the interaction of nonlinear features, no local minimum problem, no dependence on the whole data, and strong generalization ability. This paper presents a method to calculate the fixed coordinates of UWB by using a support vector machine (SVM), and gives the method and algorithm process of TDOA positioning by SVM. The simulation results show that, compared with traditional positioning algorithms such as Chan and Taylor, the proposed method is superior to the above algorithms in positioning accuracy and real-time performance. The proposed algorithm can satisfy the requirement of the positioning precision is high and the complexity of the small positioning.

\section{TDOA Principle}

TDOA refers to the mistiming of radiofrequency signals reaching two base stations. TDOA is a wireless positioning technology, which is an expression of positioning based on the difference between the distance of the radiofrequency signal reaching two base stations[8]. TDOA can be obtained by measuring the arrival time difference between mobile devices and different base stations[9]. This approach does not require strict time synchronization between the mobile device and the base station. But you have to make sure that the stations are synchronized. According to the hyperbolic algorithm, at least two intersecting hyperbolas are required for the 
position confirmation of the mobile device, and the position of the signal source is the intersection point. Therefore, at least three different base stations are needed to locate the mobile station.

In our experimental positioning system, the positioning is accomplished by the time difference between the signals of mobile devices carried by movable objects or people to different antennas. As mentioned above, the time difference between the source transmitted to different antennas at the same time is a fixed value. Next, the time difference between arrival antennas is multiplied by the speed of light to get the distance difference and hence a hyperbola. If the time difference between two base stations cannot be accurately estimated, the time difference can be measured by three or more different wireless bases. Two or more hyperbolic lines can be obtained from the measurement results and the algorithm. The intersection position of hyperbolic curves is the estimated position of the source of the mobile object. The location of the source is generally estimated in one of two ways. First, the TOA difference of the arrival time of signals of two different base stations is used. Second, the correlation processing of signals received by two different base stations is used to obtain the estimated value. [Fig. 1] shows the hyperbolic model. In our experiment, the hyperbolic model was established and solved based on the distance difference of the signal arrival, and its intersection point was the estimated position of the signal source. 
problem is transformed into a classification regression problem. Its definite process is shown in [Fig. 2].

\author{
TDOA value $\quad \begin{gathered}\text { Data } \\ \text { preprocessing }\end{gathered}$ \\ Randomly selected training set and testing set \\ Cross - validation selects the best parameter $\mathrm{c} \& \mathrm{~g}$ \\ The user location corresponding to the TDOA value \\ Classification training of SVM \\ Complete SVM classification model \\ Real-time TDOA values \\ Real-time coordinates
}




\subsection{Solution of a Classification Problem}

Support vector machines (SVM) are often used for a classification problems. The one-to-one method is to design the support vector machine by picking any two samples out of all the samples, so $\mathrm{k}$ samples need $\mathrm{k}$ times $\mathrm{k}$ minus 1 over 2 support vector machines. Finally, the method of voting selection is adopted. In this way, the multi-classification problem of $\mathrm{k}$ samples is transformed into the solution of multiple dichotomy problems. Because the one-to-one method only involves the classification of two samples, the training time can be greatly reduced. Because the number of samples of several classifiers is balanced, the classification accuracy is high. Therefore, this paper chooses the one-to-one method to classify the data set. The one-to-one classification model is used to realize the solution of coordinate values, and the classification process of coordinate solution speed is improved, as shown in [Fig. 3].

$\mathrm{SVM}_{12}$

$\begin{array}{lll}\mathrm{SVM}_{123} & \mathrm{SVM}_{13} & \text { Voting options }\end{array}$

$\mathrm{SVM}_{23}$

$\mathrm{SVM}_{1 \text {-rest }}$

$\operatorname{SVM}_{1-\text {-rest }}$

$\mathrm{SVM}_{1-\text { rest }}$

3

$\operatorname{SVM}_{1 \text {-rest }}$

2 
In the one-to-one classification of the dataset, the TDOA value is the attribute value and the output coordinate is the label value. After determining the attribute matrix and label, the classification model can be established. Take 2 sets of TDOA values at any time to determine which label it belongs to, and finally select the coordinate value with the most votes as the output. The trained classification model can be used for regression prediction, and the localization problem is transformed into a classification problem.

\section{Experimental Results}

In order to achieve the best performance of support vector machine. In this paper, the root-mean-square error and determination coefficient are compared under different quantity samples. When the quantity sample is 5000, the root-mean-square error is the lowest and the determination coefficient is close to the maximum. Therefore, the training data sample of 5000 is selected in this paper to train SVM.

The input to the vector machine algorithm supported in this paper is three TDOA values, and the coordinate value corresponding to the TDOA value is the output. In this paper, the positioning accuracy of the algorithm is analyzed from $\mathrm{X}, \mathrm{Y}$ and $\mathrm{Z} 3$ directions. The data points were collected in a three-dimensional space of $50 \mathrm{~m} \times 25 \mathrm{~m} \times 2 \mathrm{~m}$ with a sampling interval of $0.5 \mathrm{~m}$, and a total of 20,000 points were sampled. Out of 20,000 sampling points, 5000 were randomly selected as training samples.

In order to compare the positioning accuracy of this algorithm and the traditional algorithm, Chan, Taylor and the algorithm in this paper are compared under different TDOA differences. It can be seen from the [Fig. 5], Taylor algorithm is greater than the root means square error Chan algorithm. The reason is that the Taylor algorithm needs according to the initial position constantly recursion, initial estimate position had a great influence on the final results, so will cause the root mean square error is bigger. But Chan algorithm is a non-recursive hyperbolic equation solving. His algorithm complexity is smaller than Taylor, calculated two iterations can position coordinates, so Chan, root mean square error of the algorithm to be less than Taylor. As the algorithm proposed in this paper has trained the model before the output coordinates, the algorithm proposed in this paper can also achieve small root mean square error in the case of different TDOA differences.

As the number of anchor points increases, the time taken by Chan algorithm and Taylor to locate them increases. This paper compares the time taken by three algorithms at different location points. As shown in [Fig. 6], it can be seen from the figure that since the complexity 
of solving coordinates by Taylor and Chan algorithms is relatively high compared with this algorithm, the positioning time of Taylor and Chan algorithms is much longer than that of this algorithm. In this paper, support vector machine is used to calculate the TDOA of UWB. It takes some time to train the model, and the time to locate the sample points increases with the number of sample points after the model is trained. The change of positioning time is shown in [Fig. 6]. The positioning time is 1.096 seconds when the number of registration points reaches 7,000. In the case of the same positioning accuracy, the real-time performance of this algorithm is far better than that of traditional positioning algorithm.
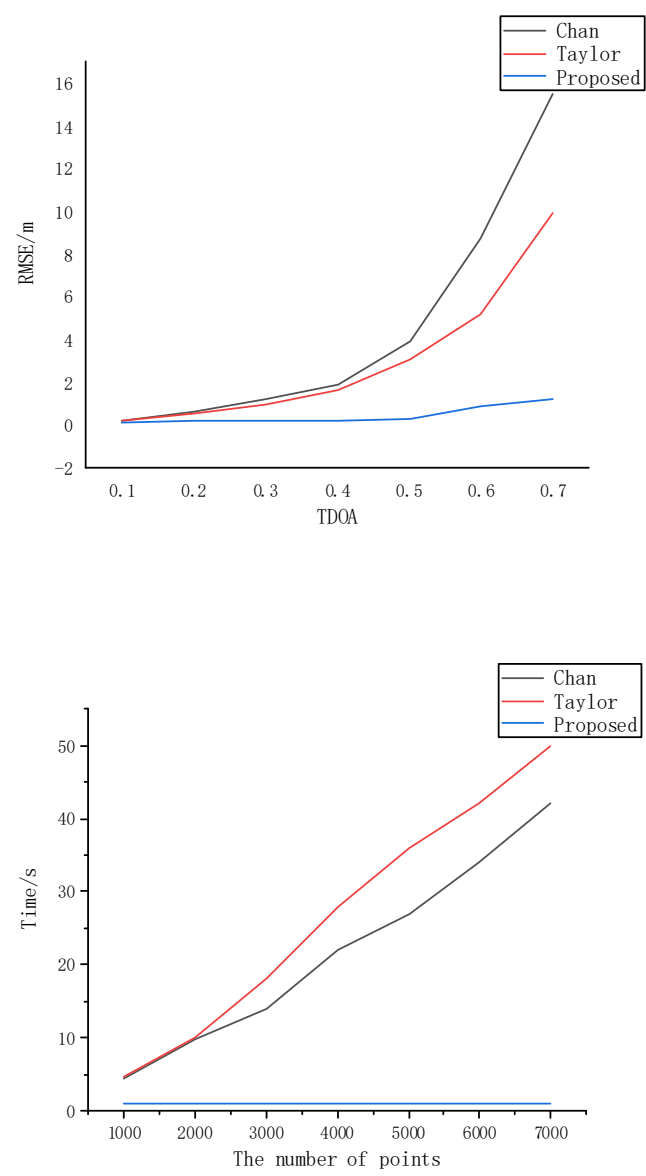
consumption of UWB in indoor high-density environment. In this paper, a UWB indoor positioning method based on SVM is proposed, and the positioning problem is transformed into the solution of multi-classification problem. Experimental results show that SVM algorithm is effective in solving the problems of large error, low refresh rate and high-power consumption of UWB in indoor positioning. The positioning accuracy and positioning time are better than the traditional algorithm. Therefore, SVM algorithm has a very wide application prospect under the application condition of high density and low power consumption in real-time positioning with positioning accuracy of centimeter.

\section{References}

[1] Zhong Y., Liu T. X., Li B. F., Yang L., Lou L., Integration of UWB and IMU for precise and continuous indoor positioning, 2018 Ubiquitous Positioning, Indoor Navigation and Location-Based Services (UPINLBS), (2018), March 22-23; Wuhan, China, DOI: 10.1109/UPINLBS.2018.8559718

[2] Zhao G., Niu H., Research and application of indoor positioning based on UWB, Third International Conference on Cyberspace Technology (CCT 2015), (2015), October 17-18; Beijing, China, DOI: $10.1049 /$ cp. 2015.0837

[3] Chan Y. T., Ho K. C., A simple and efficient estimator for hyperbolic location, IEEE Transactions on Signal Processing, (1994), Vol.42, No.8, pp.1905-1915. DOI: 10.1109/78.301830

[4] Foy W., Position-location solutions by Taylor-series estimation, IEEE Transactions on Aerospace and Electronic Systems, (1976), Vol.AES-12, No.2, pp.187-194. DOI: 10.1109/TAES.1976.308294

[5] Fang B. T., Simple solutions for hyperbolic and related position fixes, IEEE Transactions on Aerospace and Electronic Systems, (1990), Vol.26, No.5, pp.748-753. DOI: 10.1109/7.102710

[6] V. N. Koteswara, Rao. Devana, B. S. L. Mounika, B. Yamini, G. Anitha, G. BalaSaiTarun, Novel UWB Monopole Antenna with Band Notched Characteristics, International Journal of Signal Processing, Image Processing and Pattern Recognition, (2016), Vol.9, No.5, pp.291-296. DOI: 10.14257/ijsip.2016.9.5.26

[7] Ramakrishna Miryala, Vijaya Saradhi T., Combined Rule Filtering and Mapreduce Based Hadoop SVM Approach for Detecting Attacks in Big Data Security Analytics, International Journal of Database Theory and Application, (2019), Vol.12, No.4, pp.1-18. DOI: 10.33832/ijdta.2019.12.4.01

[8] Vidhya Sathish, P. Sheik Abdul Khader, Improved Detecting Host Based Intrusions Based On Hybrid SVM Using Grey Wolf Optimizer, International Journal of Security and Its Applications, (2017), Vol.11, No.9, pp.59-72. DOI: $10.14257 /$ ijsia.2017.11.9.05

[9] Guofang Dong, Bin Yang, TDOA-Based and RSSI-Based Underground Wireless Positioning Methods and Performance Analysis, International Journal of Future Generation Communication and Networking, (2015), Vol.8, No.5, pp.249-258. DOI:10.14257/ijfgcn.2015.8.5.25 